\title{
Effective velocity for transport in heterogeneous compressible flows with mean drift
}

\author{
Sabine Attinger ${ }^{1,2, a)}$ and Assyr Abdulle ${ }^{3}$ \\ ${ }^{1}$ Computational Hydrosystems, Helmholtz Centre for Environmental Research, Leipzig, Germany \\ ${ }^{2}$ Institute of Geosciences, University of Jena, Leipzig, Germany \\ ${ }^{3}$ School of Mathematics and Maxwell Institute for Mathematical Sciences, University of Edinburgh, \\ Edinburgh, United Kingdom
}

(Received 17 August 2007; accepted 13 October 2007; published online 23 January 2008)

\begin{abstract}
Solving transport equations in heterogeneous flows might give rise to scale dependent transport behavior with effective large scale transport parameters differing from those found on smaller scales. For incompressible velocity fields, homogenization methods have proven to be powerful in describing the effective transport parameters. In this paper, we aim at studying the effective drift of transport problems in heterogeneous compressible flows. Such a study was done by Vergassola and Avellaneda in Physica D 106, 148 (1997). There, it was shown that for static compressible flow without mean drift, impacts on the large scale drift do not occur. We will first discuss the impact of a mean drift and show that static compressible flow with mean drift can produce a heterogeneity driven large scale drift (or ballistic transport). For the case of Gaussian stationary random processes, we derive explicit results for the large scale drift. Moreover, we show that the large scale or effective drift depends on the small scale diffusion coefficients and thus on the molecular weights of the particles. This study could be applied to weight-based particle separation. Numerical simulations are presented to illustrate these phenomena. (C) 2008 American Institute of Physics.
\end{abstract}

[DOI: $10.1063 / 1.2827584]$

\section{INTRODUCTION}

The large-scale behavior of solute transport in heterogeneous media is a basic problem of high interest, for example, in subsurface hydrology, petroleum engineering, chemical and biomedical engineering to mention but a few. In its most general case, the velocity field in which the solute is released is taken to be incompressible, irrotational or a combination of both. Incompressible fluid flows are governed for example, in fluid dynamics by the Navier-Stokes equations or in porous media flow by the Darcian equations. Compressible flows, which might be written in form of a potential or irrotational vector fields, can arise along energy gradients in complex energy landscapes (gradient flows) or in flows with external source or sink terms.

Due to the inhomogeneities in the local flow field, the large scale transport properties are generally very different from those found on the smaller scales. A central aim is to understand the large scale behavior of transport problems governed by local inhomogeneities, for example by replacing the heterogeneous medium by an equivalent homogeneous medium with similar large scale properties. There are conceptually different methods to derive so-called homogenized system's properties as for example the homogenization theory, the stochastic modeling approach and the volume averaging approach. The idea of an equivalent homogeneous medium can be explained best by introducing the concept of a control volume $(\mathrm{CV})$ or representative volume (RV). The $\mathrm{CV}$ is chosen large enough so that a process averaged over the $\mathrm{CV}$ can be characterized by properties that are constant at

\footnotetext{
a) Author to whom correspondence should be addressed. Electronic mail: sabine.attinger@ufz.de.
}

various locations of the domain. Moreover, the CV has to be small enough to still resolve characteristic features of the process behavior in the domain. Closest to this concept is the volume averaging approach pioneered by Whitaker. ${ }^{1}$ Homogenization theory introduces two length scales, the small one corresponding to the length scale of the control volume, the large one related to the observation scale. The homogenized medium in this approach follows by asymptotic expansion. The stochastic modeling approach is first of all free of the definition of any control volume because it is based on probability theoretical concepts. Starting from a statistical description of the system behavior on a local scale with a fixed time-independent structure of the heterogeneities, the objective of the method is to derive appropriate averaged quantities characteristic for the system on length and time scales larger than the small scales. If the medium is ergodic, expectations can be replaced by averages over a representative volume over the heterogeneity, which compares with an average over the control volume. The stochastic approach is well known in solid state physics and fluid mechanics where it is used to model systems with "quenched disorder," see, e.g., the reviews by Haus and $\mathrm{Kehr}^{2}$ and Bouchaud and Georges. ${ }^{3}$ The stochastic approach has also been successfully applied to many problems in groundwater hydrology. Examples can be found in the textbooks by Dagan ${ }^{4}$ and Gelhar, to mention only a few prominent textbooks in this area.

The large scale transport behavior for solutes moving in incompressible flows has been studied by many authors. In the case of zero or weak mean divergence free flow, the large scale behavior is well understood and has been rigorously analyzed in the framework of homogenization by McLaughlin, Papanicolaou, Pironneau, ${ }^{6}$ Avellaneda and Majda, ${ }^{7}$ Ma- 
jda and Kramer, ${ }^{8}$ E, ${ }^{9}$ and many others. We refer to Ref. 8 for further references. The case of transport in divergence free flows with strong mean has been less studied and we mention Majda and MacLaughlin, ${ }^{10}$ Pavliotis ${ }^{11}$ Lunati, Attinger, and Kinzelbach, ${ }^{12}$ Abdulle and Attinger. ${ }^{13}$ We refer to Ref. 11 for further references. Only a few works have investigated solute transport in compressible flow fields (see Refs. 14-16, and the references therein).

The problem of describing the large-scale transport behavior in heterogeneous compressible flows with nonvanishing mean drift is to a large extend open and is the central focus of this paper. Using the stochastic modeling approach, we will discuss a fundamental difference between the large scale transport behavior in incompressible and compressible flows with mean drift. We study the impact of a mean drift on the effective large scale transport behavior, and show that static compressible flows can produce heterogeneity driven large scale drifts. This extends the results of Ref. 14, where is was shown that static compressible flows with a vanishing mean drift do not produce heterogeneity induced large scale drifts (or ballistic effects). Furthermore, the techniques used in this paper are different. While homogenization by multiple scale expansion was used in Ref. 14, we derive an exact formula for the large drift by means of the Green functions and Fourier techniques. Making use of perturbation theory, we present then explicit results for the effective transport velocities in compressible flows. We show that the effective drift depends on the small scale diffusion and thus on the molecular weight of the particles. This effect might be used in applications as for example for the construction of efficient sorting devices for separating macromolecules. Such devices have been proposed in Refs. 17 and 18 and are of high interest.

The paper is organized as follows: In Sec. II we formulate the transport model problem and the class of flow fields considered. In Sec. III we derive an exact theory for large scale drift and draw the correspondence with homogenization results. In Sec. IV, a perturbation expansion is given in order to obtain explicit results for the large scale drift. A qualitative study of the influence of the flow field correlation lengths and the mean velocities on the effective drifts is given. Finally in Sec. V we present numerical results illustrating the effects of the flow field correlation lengths and the mean velocities on the particle trajectories.

\section{THE TRANSPORT MODEL}

The solutes are being transported by two physical mechanisms: They are passively carried along with the fluid while spreading due to diffusion. Consequently, the time evolution of the solute concentration is described by an advection-diffusion equation with a heterogeneous flow field $\mathbf{u}(\mathbf{x})$,

$$
\begin{aligned}
& \frac{\partial}{\partial t} c(\mathbf{x}, t)+\nabla \cdot[\mathbf{u}(\mathbf{x}) c(\mathbf{x}, t)]-\nabla \cdot[\mathbf{D} \nabla c(\mathbf{x}, t)]=0, \\
& \left.\quad c\right|_{t=0}=\rho(\mathbf{x}),
\end{aligned}
$$

where $c(\mathbf{x}, t)$ is the particle concentration and $\mathbf{D}$ is the diffusion tensor. We assume $\mathbf{x} \in \mathbb{R}^{d}, d=2,3$ and vanishing concentrations at infinity as boundary conditions. We decompose the velocity field into a constant mean value $\overline{\mathbf{u}}$ and a fluctuating part $\widetilde{\mathbf{u}}(\mathbf{x})$ around this mean value,

$$
\mathbf{u}(\mathbf{x}) \equiv \overline{\mathbf{u}}+\widetilde{\mathbf{u}}(\mathbf{x}) .
$$

In its most general form, any flow field $\widetilde{\mathbf{u}}(\mathbf{x})$ is composed of an incompressible and an irrotational part,

$$
\widetilde{\mathbf{u}}(\mathbf{x})=\mathbf{v}(\mathbf{x})+\mathbf{w}(\mathbf{x}),
$$

with $\nabla \cdot \mathbf{v}(\mathbf{x}) \equiv 0$ and $\nabla \times \mathbf{w}(\mathbf{x}) \equiv 0$, respectively. Both parts, the incompressible as well as the irrotational one, may be generated by so-called potentials. Any incompressible velocity field has a vector potential $\mathbf{A}(\mathbf{x})$ such that

$$
\mathbf{v}(\mathbf{x})=\nabla \times \mathbf{A}(\mathbf{x}) .
$$

Note that an incompressible field cannot be described as having a scalar potential in general. This is only the case for any irrotational field which can always be expressed (for a simply connected domain as we consider here) by some potential $\phi$ such that

$$
\mathbf{w}(\mathbf{x})=-\nabla \phi(\mathbf{x}) .
$$

\section{A. Transport parameters}

In uniform flow fields, the transport velocity $u_{j}(t)$ and the dispersion coefficients $D_{i j}(t)$ are given by

$$
\begin{aligned}
& u_{j}(t)=\frac{\mathrm{d}}{\mathrm{d} t} m_{j}^{(1)}(t), \\
& D_{i j}(t)=\frac{1}{2} \frac{\mathrm{d}}{\mathrm{d} t}\left[m_{i j}^{(2)}(t)-m_{i}^{(1)}(t) m_{j}^{(1)}(t)\right],
\end{aligned}
$$

where $m_{j}^{(1)}(t)$ and $m_{i j}^{(2)}(t)$ are the first two moments of the properly normalized spatial concentration distribution in $d$ dimensions,

$$
\begin{aligned}
& m_{j}^{(1)}(t)=\int \mathrm{d}^{d} x x_{j} p(\mathbf{x}, t), \\
& m_{i j}^{(2)}(t)=\int \mathrm{d}^{d} x x_{i} x_{j} p(\mathbf{x}, t),
\end{aligned}
$$

with a density $p(\mathbf{x}, t)$,

$$
p(\mathbf{x}, t)=\frac{c(\mathbf{x}, t)}{\int \mathrm{d}^{d} y c(\mathbf{y}, t)} .
$$

Since the total mass $\int \mathrm{d}^{d} y c(\mathbf{y}, t)$ is conserved for all times and equal to a constant, in the following we will set it to one, and thus identify density $p(\mathbf{x}, t)$ with concentration $c(\mathbf{x}, t)$.

For heterogeneous flow fields, transport properties are generally very different from those found in uniform flows. In the following, we describe the stochastic approach for the study of such flow fields. Starting from a statistical description of the system behavior on a local scale with a fixed time-independent structure of the heterogeneities, the objective is to derive equivalent quantities, characteristic for the 
system on larger length and time scales. In the stochastic modeling approach, the given heterogeneous medium is identified as one particular typical realization of a spatial stochastic process. The basic assumption of the approach is that the transport coefficients can be expressed as averages over the ensemble of all possible mediums realizations denoted by the overbar in the following expression:

$$
\begin{aligned}
& u_{j}^{\text {ens }}(t)=\frac{\mathrm{d}}{\mathrm{d} t} \overline{m_{j}^{(1)}(t)}, \\
& D_{i j}^{\text {ens }}(t)=\frac{1}{2} \frac{\mathrm{d}}{\mathrm{d} t}\left[\overline{m_{i j}^{(2)}(t)}-\overline{m_{i}^{(1)}(t) m_{j}^{(1)}(t)}\right] .
\end{aligned}
$$

By construction, such averages represent properties characteristic for the whole ensemble of all possible mediums realizations. On the first sight, the stochastic approach might seem to be of limited value to predict transport properties for a single given medium. The reason why the stochastic method does have predictive power also for the individual medium lies in the fact that for appropriate quantities, the fluctuations from realization to realization become small after the concentration distribution has sampled a sufficiently large representative part of the given medium. The characteristic transport properties found in different realizations of the medium then fluctuate only weakly around the ensemble averages constructed in the stochastic approach, so the aforementioned properties represent realistic values typically found at large-scales in one given heterogeneous medium. In the limit $t \rightarrow \infty$, the ensemble averaged quantities converge to the large scale quantities of the single realization. ${ }^{4,5}$ These asymptotic transport parameters are called effective parameters in the following:

$$
\begin{aligned}
& u_{j}^{\mathrm{eff}} \equiv u_{j}^{\mathrm{ens}}(t=\infty), \\
& D_{i j}^{\mathrm{eff}} \equiv D_{i j}^{\mathrm{ens}}(t=\infty) .
\end{aligned}
$$

They are equivalent to transport parameters derivable in homogenization theory, when this latter theory applies.

\section{B. Fourier transform}

To study the transport parameters-transport velocity and dispersion coefficients - it is preferable to transform the transport equation (1) into Fourier variables. We define

$$
\begin{aligned}
& \hat{c}(\mathbf{q}, t)=\int \mathrm{d}^{d} x c(\mathbf{x}, t) \exp (+i \mathbf{q} \mathbf{x}) \quad \text { and } \\
& c(\mathbf{x}, t)=\int_{\mathbf{q}} \hat{c}(\mathbf{q}, t) \exp (-i \mathbf{q} \mathbf{x}),
\end{aligned}
$$

where $\hat{c}(\mathbf{q}, t)$ denotes the Fourier transform of $c$ and where $\mathbf{q}, \mathbf{x} \in \mathbb{R}^{d}$ are $d$-dimensional vectors and $\mathbf{q x}$ denotes the corresponding scalar product. For the $d$-dimensional q-integration over the whole space here and in the following we employ the short-hand notation:

$$
\int_{\mathbf{q}} \ldots \equiv \int \frac{\mathrm{d}^{d} q}{(2 \pi)^{d}} \ldots
$$

The transport Eq. (1) then reads in Fourier variables

$$
\begin{aligned}
& \frac{\partial}{\partial t} \hat{c}(\mathbf{q}, t)+(-\mathrm{i} \mathbf{q} \overline{\mathbf{u}}+\mathbf{q} \mathbf{D q}) \hat{c}(\mathbf{q}, t)-\int_{\mathbf{q}^{\prime}} i \mathbf{q} \hat{\tilde{\mathbf{u}}}\left(\mathbf{q}-\mathbf{q}^{\prime}\right) \hat{c}\left(\mathbf{q}^{\prime}, t\right) \\
& \quad=\delta(t) \hat{\rho}(\mathbf{q}) .
\end{aligned}
$$

For short hand notation, we define the following operators:

$$
\begin{aligned}
& \mathcal{R}\left(\mathbf{q}, \mathbf{q}^{\prime}\right) \equiv i \mathbf{q} \cdot \hat{\tilde{\mathbf{u}}}\left(\mathbf{q}-\mathbf{q}^{\prime}\right), \\
& L(\mathbf{q} ; t) \cdot \equiv \frac{\partial \cdot}{\partial t}+(-i \mathbf{q} \overline{\mathbf{u}}+\mathbf{q} D \mathbf{q}) \cdot .
\end{aligned}
$$

We denote by $\hat{c}_{0}(\mathbf{q}, t)$ the solution of the problem $L(\mathbf{q} ; t) \hat{c}_{0}(\mathbf{q}, t)=\delta(t)$. It is given by

$$
\hat{c}_{0}(\mathbf{q}, t)=\Theta(t) \exp [-(\mathbf{q} \mathbf{D q}-\mathrm{i} \overline{\mathbf{u}} \mathbf{q}) t] .
$$

Here and in what follows, we denote by $\Theta(\cdot)$ the Heaviside function $[\Theta(t)=1$ if $t \geqslant 0,0$ otherwise]. For simplicity we assume a point-like injection as an initial concentration in Eq. (2), i.e., $\rho(\mathbf{x})=\delta(\mathbf{x})$, which reduces to $\hat{\rho}(\mathbf{k})=1$ in Fourier variables. We can then rewrite Eq. (18) as an integral equation,

$$
\hat{c}(\mathbf{q}, t)=\hat{c}_{0}(\mathbf{q}, t)+\int_{-\infty}^{\infty} \mathrm{d} t^{\prime} \hat{c}_{0}\left(\mathbf{q}, t-t^{\prime}\right) \int_{\mathbf{q}^{\prime}} \mathcal{R}\left(\mathbf{q}, \mathbf{q}^{\prime}\right) \hat{c}\left(\mathbf{q}^{\prime}, t^{\prime}\right) .
$$

From the definitions of transport velocity and the dispersion coefficients in one single realization as given by Eqs. (7) and (8), one gets the following equivalent representation in the Fourier space:

$$
\begin{aligned}
& u_{j}(t)=\left.\frac{\mathrm{d}}{\mathrm{d} t}\left(-\mathrm{i} \partial_{q_{j}}\right)[\ln \hat{c}(\mathbf{q}, t)]\right|_{\mathbf{q}=0}, \\
& D_{i j}(t)=\left.\frac{1}{2} \frac{\mathrm{d}}{\mathrm{d} t}\left(-\mathrm{i} \partial_{q_{i}}\right)\left(-i \partial_{q_{j}}\right)[\ln \hat{c}(\mathbf{q}, t)]\right|_{\mathbf{q}=0},
\end{aligned}
$$

where $\partial_{q_{i}}$ denotes the partial derivative with respect to the q-component in the $i$-direction. The ensemble averaged quantities defined by Eqs. (12) and (13) now read

$$
\begin{aligned}
& u_{j}^{\mathrm{ens}}(t)=\left.\frac{\mathrm{d}}{\mathrm{d} t}\left(-\mathrm{i} \partial_{q_{j}}\right)[\ln \overline{\hat{c}(\mathbf{q}, t)}]\right|_{\mathbf{q}=0}, \\
& D_{i j}^{\mathrm{ens}}(t)=\left.\frac{1}{2} \frac{\mathrm{d}}{\mathrm{d} t}\left(-\mathrm{i} \partial_{q_{i}}\right)\left(-i \partial_{q_{j}}\right)[\ln \overline{\hat{c}(\mathbf{q}, t)}]\right|_{\mathbf{q}=0} .
\end{aligned}
$$

\section{The flow field}

We consider the velocity field as introduced in Eq. (3) and define the fluctuating field $\widetilde{\mathbf{u}}$ as a zero mean Gaussian stationary random process with a correlation function given by 


$$
\overline{\tilde{u}_{i}(\mathbf{x}) \tilde{u}_{j}\left(\mathbf{x}^{\prime}\right)}=C_{i j}\left(\mathbf{x}, \mathbf{x}^{\prime}\right)
$$

Owing to the stationarity assumption, the correlations are translation invariant and depend only on the difference between their arguments. Then, the correlation functions of the Fourier transformed velocity fluctuations read

$$
\overline{\hat{\tilde{u}}_{i}(\mathbf{q}) \hat{\tilde{u}}_{j}\left(\mathbf{q}^{\prime}\right)}=(2 \pi)^{d} \hat{C}_{i j}(\mathbf{q}) \delta\left(\mathbf{q}+\mathbf{q}^{\prime}\right)
$$

By employing the velocity decomposition (4), the velocity correlations consist of the two parts,

$$
\hat{C}_{i j}(\mathbf{q})=\hat{C}_{i j}^{v v}(\mathbf{q})+\hat{C}_{i j}^{w w}(\mathbf{q})
$$

where the upper index refer to the contribution originating from the incompressible and the irrotational velocity fields $\mathbf{v}$ and $\mathbf{w}$, respectively.

Our main interest is in studying the influence of irrotational flows upon large scale transport. Thus, we neglect in the following the incompressible part in the flow field and assume

$$
\hat{C}_{i j}(\mathbf{q})=\hat{C}_{i j}^{w w}(\mathbf{q})
$$

For the correlation function of the irrotational flow fields, we choose

$$
\hat{C}_{i j}(\mathbf{q})=-q_{i} q_{j} \gamma(\mathbf{q})
$$

where $\gamma(\mathbf{q})$ is given by a Gauss-shaped function

$$
\gamma(\mathbf{q})=\sigma^{2}(2 \pi)^{d / 2} \prod_{i=1}^{d} l_{i} \exp \left[-\left(q_{i} l_{i}\right)^{2} / 2\right] .
$$

The length scales $l_{i}$ are the correlation lengths in the $i$-direction (with $i=1 \ldots d$ in a $d$-dimensional system) and $\sigma^{2}$ is the variance.

\section{EXACT THEORY FOR LARGE SCALE DRIFT}

In the following, we will concentrate on the large scale drift. The calculation of large scale dispersion coefficients is beyond the scope of this paper. Starting from the transport equation (1) we first introduce the Green function and its adjoint. By making use of the translation invariance of the averaged Green function and its adjoint, we derive an exact and closed formula for the ensemble averaged concentration distribution and for the large scale transport velocity.

\section{A. Green function and its adjoint}

The Green function of the transport equation (1) is defined as the function which solves

$$
\begin{gathered}
\frac{\partial}{\partial t} g\left(\mathbf{x}, \mathbf{x}^{\prime}, t\right)+\nabla\left[\mathbf{u}(\mathbf{x}) g\left(\mathbf{x}, \mathbf{x}^{\prime}, t\right)\right]-\nabla \mathbf{D} \nabla g\left(\mathbf{x}, \mathbf{x}^{\prime}, t\right) \\
=\delta^{d}\left(\mathbf{x}-\mathbf{x}^{\prime}\right) \delta(t),
\end{gathered}
$$

where the delta functions $\delta^{d}\left(\mathbf{x}-\mathbf{x}^{\prime}\right) \delta(t)$ represent an instantaneous injection at $t=0$ for $\mathbf{x}=\mathbf{x}^{\prime}$.

The equivalent integral equation in the Fourier space then reads

$$
\begin{aligned}
\hat{g}\left(\mathbf{q}, \mathbf{q}^{\prime}, t\right)= & (2 \pi)^{d} \delta\left(\mathbf{q}+\mathbf{q}^{\prime}\right) \hat{g}_{0}(\mathbf{q}, t)+\int_{-\infty}^{\infty} \mathrm{d} t^{\prime} \hat{g}_{0}\left(\mathbf{q}, t-t^{\prime}\right) \\
& \times \int_{\mathbf{q}^{\prime \prime}} \mathcal{R}\left(\mathbf{q}, \mathbf{q}^{\prime \prime}\right) \hat{g}\left(\mathbf{q}^{\prime \prime}, \mathbf{q}^{\prime}, t^{\prime}\right),
\end{aligned}
$$

where $g_{0}=c_{0}$ as defined previously. We will need in the following the adjoint Green function $g^{+}\left(x, x^{\prime}, t\right)$, defined as the function which solves the adjoint equation

$$
\begin{aligned}
& \frac{\partial}{\partial t} g^{+}\left(\mathbf{x}, \mathbf{x}^{\prime}, t\right)-\mathbf{u}(\mathbf{x}) \nabla g^{+}\left(\mathbf{x}, \mathbf{x}^{\prime}, t\right)-\nabla \mathbf{D} \nabla g^{+}\left(\mathbf{x}, \mathbf{x}^{\prime}, t\right) \\
& \quad=\delta^{d}\left(\mathbf{x}-\mathbf{x}^{\prime}\right) \delta(t) .
\end{aligned}
$$

Its corresponding integral equation in the Fourier space reads

$$
\begin{aligned}
\hat{g}^{+}\left(\mathbf{q}, \mathbf{q}^{\prime}, t\right)= & (2 \pi)^{d} \delta\left(\mathbf{q}+\mathbf{q}^{\prime}\right) \hat{g}_{0}^{+}(\mathbf{q}, t)+\int_{-\infty}^{\infty} \mathrm{d} t^{\prime} \hat{g}_{0}^{+}\left(\mathbf{q}, t-t^{\prime}\right) \\
& \times \int_{\mathbf{q}^{\prime \prime}} \mathcal{R}^{+}\left(\mathbf{q}, \mathbf{q}^{\prime \prime}\right) \hat{g}^{+}\left(\mathbf{q}^{\prime \prime}, \mathbf{q}^{\prime}, t^{\prime}\right)
\end{aligned}
$$

where $\mathcal{R}^{+}\left(\mathbf{q}, \mathbf{q}^{\prime \prime}\right)=-i \mathbf{q}^{\prime \prime} \hat{\tilde{\mathbf{u}}}\left(\mathbf{q}-\mathbf{q}^{\prime \prime}\right)$. With the aid of the generalized Green theorem, the Green function and its adjoint are related by (see Ref. 20, Chap. 7)

$$
g^{+}\left(\mathbf{x}, \mathbf{x}^{\prime}, t\right)=g\left(\mathbf{x}^{\prime}, \mathbf{x}, t\right),
$$

which reads in Fourier space

$$
\hat{g}^{+}\left(\mathbf{q}, \mathbf{q}^{\prime}, t\right)=\hat{g}\left(\mathbf{q}^{\prime}, \mathbf{q}, t\right)=\hat{g}\left(-\mathbf{q}^{\prime},-\mathbf{q}, t\right)
$$

According to Ref. 22 the ensemble averaged Green function and its adjoint are translation invariant,

$$
\overline{g^{+}\left(\mathbf{x}, \mathbf{x}^{\prime}, t\right)}=\overline{g\left(\mathbf{x}^{\prime}, \mathbf{x}, t\right)}=\overline{g\left(\mathbf{x}^{\prime}-\mathbf{x}, t\right)},
$$

where $g\left(\mathbf{x}^{\prime}-\mathbf{x}, t\right)=g\left(\mathbf{x}^{\prime}-\mathbf{x}, 0, t\right)$. From this last relation, we derive in Fourier space

$$
\overline{\hat{g}\left(\mathbf{q}, \mathbf{q}^{\prime}, t\right)}=(2 \pi)^{d} \delta\left(\mathbf{q}+\mathbf{q}^{\prime}\right) \overline{\hat{g}(\mathbf{q}, t)} \text {. }
$$

The relations between the Green function and its adjoint will be used for the derivation of the closed formula of the ensemble averaged concentration distribution in the next subsection. 


\section{B. Ensemble averaged concentration distribution}

In order to derive the ensemble averaged concentration distribution, we start from the ensemble averaged Fourier transformed Green function,

$$
\begin{aligned}
\overline{\hat{g}\left(\mathbf{q}, \mathbf{q}^{\prime}, t\right)}= & (2 \pi)^{d} \delta\left(\mathbf{q}+\mathbf{q}^{\prime}\right) \hat{g}_{0}(\mathbf{q}, t)+\int_{-\infty}^{\infty} \mathrm{d} t^{\prime} \hat{g}_{0}\left(\mathbf{q}, t-t^{\prime}\right) \\
& \times \int_{\mathbf{q}^{\prime \prime}} i \mathbf{q} \overline{\hat{\tilde{\mathbf{u}}}\left(\mathbf{q}-\mathbf{q}^{\prime \prime}\right) \hat{g}\left(\mathbf{q}^{\prime \prime}, \mathbf{q}^{\prime}, t^{\prime}\right)}
\end{aligned}
$$

The Fourier transformed concentration $\hat{c}(\mathbf{q}, t)$ follows from the Green function (33) by

$$
\hat{c}(\mathbf{q}, t)=\int_{\mathbf{q}^{\prime}} \hat{g}\left(\mathbf{q}, \mathbf{q}^{\prime}, t\right) .
$$

Since the ensemble averaged Green function is translation invariant, according to Eq. (40) the right-hand side has to be proportional to $\delta\left(\mathbf{q}+\mathbf{q}^{\prime}\right)$ and we obtain

$$
\begin{aligned}
\overline{\hat{c}(\mathbf{q}, t)} & =\hat{c}_{0}(\mathbf{q}, t)+\int_{-\infty}^{\infty} \mathrm{d} t^{\prime} \hat{g}_{0}\left(\mathbf{q}, t-t^{\prime}\right) \int_{\mathbf{q}^{\prime \prime}} i \overline{\mathbf{q}} \overline{\tilde{\tilde{\mathbf{u}}}\left(\mathbf{q}-\mathbf{q}^{\prime \prime}\right) \int_{\mathbf{q}^{\prime}} \hat{g}\left(\mathbf{q}^{\prime \prime}, \mathbf{q}^{\prime}, t^{\prime}\right)} \\
& =\hat{c}_{0}(\mathbf{q}, t)+\int_{-\infty}^{\infty} \mathrm{d} t^{\prime} \hat{g}_{0}\left(\mathbf{q}, t-t^{\prime}\right) \int_{\mathbf{q}^{\prime \prime}} i \mathbf{q} \overline{\tilde{\mathbf{u}}\left(\mathbf{q}-\mathbf{q}^{\prime \prime}\right) \int_{\mathbf{q}^{\prime}} \hat{g}\left(\mathbf{q}^{\prime \prime},-\mathbf{q}, t^{\prime}\right)} \\
& =\hat{c}_{0}(\mathbf{q}, t)+\int_{-\infty}^{\infty} \mathrm{d} t^{\prime} \hat{g}_{0}\left(\mathbf{q}, t-t^{\prime}\right) \int_{\mathbf{q}^{\prime \prime}} i \mathbf{q} \overline{\tilde{\mathbf{u}}\left(\mathbf{q}-\mathbf{q}^{\prime \prime}\right) \int_{\mathbf{q}^{\prime}} \hat{g}^{+}\left(-\mathbf{q}^{\prime},-\mathbf{q}^{\prime \prime}, t^{\prime}\right) .}
\end{aligned}
$$

We identified $\hat{g}\left(\mathbf{q}^{\prime}, \mathbf{q}^{\prime \prime}, t^{\prime}\right)$ with the Fourier transformed adjoint Green function, $\hat{g}^{+}\left(-\mathbf{q}^{\prime \prime},-\mathbf{q}^{\prime}, t^{\prime}\right)$ according to Eq. (38). Finally we replace the adjoint Green function by its integral equation (36) and get

$$
\begin{aligned}
\overline{\hat{c}(\mathbf{q}, t)} & =\hat{c}_{0}(\mathbf{q}, t)+\int_{-\infty}^{\infty} \mathrm{d} t^{\prime} \int_{-\infty}^{\infty} \mathrm{d} t^{\prime \prime} \hat{g}_{0}\left(\mathbf{q}, t-t^{\prime}\right) \hat{g}_{0}\left(\mathbf{q}, t^{\prime}-t^{\prime \prime}\right) \int_{\mathbf{q}^{\prime \prime}} \int_{\mathbf{q}^{\prime \prime \prime}} i \overline{\mathbf{q} \hat{\tilde{\mathbf{u}}}\left(\mathbf{q}-\mathbf{q}^{\prime \prime}\right) i\left(\mathbf{q}^{\prime \prime \prime}\right) \hat{\tilde{\mathbf{u}}}\left(\mathbf{q}^{\prime}+\mathbf{q}^{\prime \prime \prime}\right) \int_{\mathbf{q}^{\prime}} \hat{g}^{+}\left(\mathbf{q}^{\prime \prime \prime},-\mathbf{q}^{\prime \prime}, t^{\prime \prime}\right)} \\
& =\hat{c}_{0}(\mathbf{q}, t)+\int_{-\infty}^{\infty} \mathrm{d} t^{\prime} \int_{-\infty}^{\infty} \mathrm{d} t^{\prime \prime} \hat{g}_{0}\left(\mathbf{q}, t-t^{\prime}\right) \hat{g}_{0}\left(\mathbf{q}, t^{\prime}-t^{\prime \prime}\right) \int_{\mathbf{q}^{\prime \prime}} \int_{\mathbf{q}^{\prime \prime \prime}} i \mathbf{q} \hat{\tilde{\mathbf{u}}}\left(\mathbf{q}^{\prime \prime}\right) i\left(\mathbf{q}+\mathbf{q}^{\prime \prime \prime}\right) \hat{\tilde{\mathbf{u}}}\left(\mathbf{q}^{\prime \prime \prime}\right) \int_{\mathbf{q}^{\prime}} \hat{g}\left(\mathbf{q}-\mathbf{q}^{\prime \prime},-\mathbf{q}-\mathbf{q}^{\prime \prime \prime}, t^{\prime \prime}\right) .
\end{aligned}
$$

\section{Large scale drift}

We can now derive the exact large scale drift defined as the limit of $\mathbf{u}^{\text {ens }}$ [see Eq. (25)] when $t \rightarrow \infty$,

$$
u_{j}^{\mathrm{eff}} \equiv \lim _{t \rightarrow \infty} u_{j}^{\mathrm{ens}}(t)=\left.\frac{\mathrm{d}}{\mathrm{d} t}\left(-\mathrm{i} \partial_{q_{j}}\right)[\ln \overline{\hat{c}(\mathbf{q}, t=\infty)}]\right|_{\mathbf{q}=0} .
$$

By using Eq. (44), we obtain

$$
u_{j}^{\text {eff }}=\bar{u}_{j}+\int_{-\infty}^{\infty} \mathrm{d} t^{\prime} \int_{\mathbf{q}^{\prime}} \int_{\mathbf{q}^{\prime \prime}} \overline{\hat{\tilde{u_{j}}}\left(\mathbf{q}^{\prime}\right) i \mathbf{q}^{\prime \prime} \hat{\tilde{\mathbf{u}}}\left(\mathbf{q}^{\prime \prime}\right) \hat{g}\left(-\mathbf{q}^{\prime},-\mathbf{q}^{\prime \prime}, t^{\prime}\right)},
$$

where we changed the variables in Eq. (46) according to $t^{\prime \prime}$ $\rightarrow t^{\prime}, \mathbf{q}^{\prime \prime \prime} \rightarrow \mathbf{q}^{\prime \prime}, \mathbf{q}^{\prime \prime} \rightarrow \mathbf{q}^{\prime}$.

If the flow field is incompressible, $u_{j}^{\text {eff }}$ reduces to $\bar{u}_{j}$ since the assumption $\nabla \cdot \mathbf{u}=0$ translates in Fourier space into $\mathbf{q} \cdot \mathbf{u}$ $=\Sigma_{i} q_{i} u_{i}=0$ and the integrand in the integral in Eq. (45) vanishes. Thus, the incompressible parts of heterogeneous flow fields show no impact on the effective large scale drift of the particles. Only the irrotational parts of a flow field can contribute to an effective drift. Since the Green function $g$ depends on small scale diffusive movements of the particles, the effective drift $u_{j}^{\text {eff }}$ becomes a function of the small scale diffusion as well, i.e., $u_{j}^{\text {eff }}=u_{j}^{\text {eff }}(D)$.

\section{Comparison with homogenization theory results}

In the following, we show that the velocity components can be represented by

$$
u_{j}^{\text {eff }}=\bar{u}_{j}+\int_{-\infty}^{\infty} \mathrm{d} t^{\prime} \overline{\tilde{u_{j}}(\mathbf{x}) \psi\left(\mathbf{x}, t^{\prime}\right)}
$$

where the auxiliary function $\psi(\mathbf{x}, t)$ is the solution of the equation

$$
\begin{aligned}
\frac{\partial}{\partial t} \psi(\mathbf{x}, t) & +\nabla \cdot[\mathbf{u}(\mathbf{x}) \psi(\mathbf{x}, t)]-\nabla \cdot[\mathbf{D} \nabla \psi(\mathbf{x}, t)] \\
= & -\nabla \widetilde{\mathbf{u}}(\mathbf{x}) \delta(t) .
\end{aligned}
$$

The solvability conditions $\overline{\nabla \widetilde{\mathbf{u}}}=0$ is satisfied, so that Eq. (48) has a solution (see Ref. 14). To impose unicity we require 
$\overline{\psi(\mathbf{x}, t)}=0$. This type of representation is known in homogenization theory and can be derived by a two-scale analysis. ${ }^{14}$ Here we give a different derivation for Eq. (47). With the aid of the Green function (33) we can write the $\psi$ as

$$
\hat{\psi}(-\mathbf{q}, t)=-\int_{\mathbf{q}^{\prime \prime}} i \mathbf{q}^{\prime \prime} \hat{\tilde{\mathbf{u}}}\left(\mathbf{q}^{\prime \prime}\right) \hat{g}\left(-\mathbf{q},-\mathbf{q}^{\prime \prime}, t\right),
$$

since the Fourier back-transform of $\hat{\psi}(\mathbf{q}, t)$ is given by

$$
\psi(\mathbf{x}, t)=-\int d^{d} x^{\prime} \nabla \cdot \widetilde{\mathbf{u}}\left(\mathbf{x}^{\prime}\right) g\left(\mathbf{x}, \mathbf{x}^{\prime}, t\right)
$$

With help of the above representation of $\psi$, the ensemble velocity components (45) read

$$
\begin{aligned}
u_{j}^{\text {eff }}= & \bar{u}_{j}+\int_{-\infty}^{\infty} \mathrm{d} t^{\prime} \int_{\mathbf{q}} \overline{\hat{\tilde{u_{j}}}\left(\mathbf{q}^{\prime}\right) \hat{\psi}\left(-\mathbf{q}^{\prime}, t^{\prime}\right)}=\bar{u}_{j} \\
& +\int_{-\infty}^{\infty} \mathrm{d} t^{\prime} \overline{\tilde{u_{j}}(\mathbf{x}) \psi\left(\mathbf{x}, t^{\prime}\right)}
\end{aligned}
$$

Using this equation, expression (46) can be rewritten as Eq. (47).

To establish the link to results of Ref. 14 we add to $\psi$ the constant value one defined as $\bar{\psi}$,

$$
\psi^{\prime} \equiv \psi+1 \equiv \psi+\bar{\psi}
$$

Making use of the new variable $\psi^{\prime}$ we can write Eq. (48) as

$$
\frac{\partial}{\partial t} \psi^{\prime}(\mathbf{x}, t)+\nabla\left[\mathbf{u}(\mathbf{x}) \psi^{\prime}(\mathbf{x}, t)\right]-\nabla \mathbf{D} \nabla \psi^{\prime}(\mathbf{x}, t)=0
$$

with $\overline{\psi^{\prime}(\mathbf{x}, t)}=1$. An exact solution for this differential equation without mean drift is given by the normalized Boltzmann distribution ${ }^{14}$

$$
\psi^{\prime}=\exp (-\phi / D) / \overline{\exp (-\phi / D)},
$$

where $-\nabla \phi(\mathbf{x})=\mathbf{u}(\mathbf{x})$. Making use of this solution, Vergassola and Avellaneda ${ }^{14}$ showed that static potential flows cannot produce any heterogeneity induced large scale drift components, and that the large scale drift equals the local drift value. The assumption of a vanishing mean drift is crucial for this statement to hold. In this latter case, the normalized Boltzmann distribution solves Eq. (53). For heterogeneous static potential flows with local mean drift, the normalized Boltzmann distribution (54) does not solve Eq. (53) which can be checked by inserting Eq. (54) into Eq. (53). We wish to emphasize that in Ref. 14 the hypothesis of stationarity for the velocity field has to be made after the Galilean transformation is performed and thus the discussion in Ref. 14 is restricted to velocity field without mean drift. Our aim in the next sections is to give explicit results for the large scale drift components of transport in potential flows with nonvanishing mean drift. We will demonstrate that in this case, nontrivial heterogeneities induced large scale drift appears.

\section{PERTURBATION THEORY FOR LARGE SCALE DRIFT}

Generally, there is no closed solution for Eq. (44) and therefore, for the transport parameter $u_{i}^{\text {eff }}$ for a given flow field $\widetilde{\mathbf{u}}$. To derive explicit results for the effective drift, we employ perturbation theory. Such techniques, pioneered by Kraichnan $^{23}$ and Roberts ${ }^{24}$ for turbulent diffusion, have been widely used by many authors. We construct an approximation of Eq. (44) by inserting the implicit solution and iterating the procedure. We truncate the obtained expression after the two first terms and insert it in Eq. (12) (see for example, Ref. 5 for more details on perturbation theory). Eventually, one performs the ensemble averages using the correlation functions (27). One ends up with explicit integral expressions for the quantities under consideration,

$$
u_{j}^{\mathrm{eff}}(t)=\bar{u}_{j}+\delta u_{j}^{\mathrm{ens}}(t=\infty),
$$

where $\bar{u}_{j}$ is the local value and $\delta u_{j}^{\text {ens }}(t=\infty)$ is a perturbation, reflecting the influence of the spatial heterogeneities.

In second order approximation, the integral expressions for the heterogeneity induced transport velocity components $\delta u_{j}^{\text {eff }}$ are derived as follows:

$$
\begin{aligned}
\delta u_{j}^{\text {eff }} & =\int_{-\infty}^{\infty} \mathrm{d} t^{\prime} \int_{\mathbf{q}^{\prime}} \int_{\mathbf{q}^{\prime \prime}} \overline{\tilde{u_{j}}}\left(\mathbf{q}^{\prime}\right) i \mathbf{q}^{\prime \prime} \widetilde{\mathbf{u}}\left(\mathbf{q}^{\prime \prime}\right) g\left(-\mathbf{q}^{\prime},-\mathbf{q}^{\prime \prime}, t^{\prime}\right) \\
& =-\sum_{j} \int_{0}^{\infty} d t^{\prime} \int_{\mathbf{q}^{\prime}} i q_{j}^{\prime} \hat{C}_{i j}\left(\mathbf{q}^{\prime}\right) \hat{g}_{0}\left(-\mathbf{q}^{\prime}, t^{\prime}\right)
\end{aligned}
$$

where we used the correlation function (31) and replaced $\int_{\infty}^{\infty}$ with $\int_{0}^{\infty}$ since $\hat{g}_{0}$ vanishes for $t^{\prime} \leqslant 0$ [see Eq. (21)].

As in the exact theory, $\delta u^{\text {ens }}$ vanishes for purely incompressible velocity fields since $\sum_{j} i q_{j} C_{i j}^{v v}(\mathbf{q})$ is zero for incompressible velocities and the integral in Eq. (56) vanishes. Our main interest in the following is in studying the influence of irrotational flows upon large scale transport.

\section{A. Results}

The effective drift depends on the small scale diffusion coefficient (since the Green function does) and thus on the molecular weights of the particles. This effect might be used to construct efficient sorting devices that separate macromolecules according to their molecular weights. Such devices have been proposed in Refs. 17 and 18 and are of high interest.

Advective and diffusive movement of the particles might be compared by introducing a quantity called Peclet number, $\mathrm{Pe} \equiv \bar{u} l / D$, where $\bar{u}$ is the absolute value of a typical velocity, $l$ is a typical system length (in our case the correlation length), and $D$ is the diffusion coefficient.

In the sequel we study the effect of the heterogeneities on the effective drift behavior. We will see that transport is strongly influenced by the heterogeneous structure of the medium resulting in a deviation of particles from the mean streamline $\bar{u}$ and thus in an effective drift term $\delta u_{j}^{\text {eff }}$.

Remark 1: Evaluating the integrals in Eq. (56) for diffusion dominated transport, $\delta u_{j}^{e f f}$ turns out to be proportional to $1 / D$ and, thus, the impact of the heterogeneities decreases with increasing diffusion coefficients. In the limit of vanish- 
ing Peclet numbers, the effective drift and effective mixing coefficients reduce to their local values. This finding is consistent with the results of Vergassola and Avellaneda, ${ }^{14}$ who found that transport in compressible flows with vanishing mean drift do not induce large scale velocities. Notice that vanishing Peclet numbers is equivalent to the assumption of a neglectible mean drift.

\section{B. Large scale velocity components}

Inserting the explicit form of the velocity correlation function (32) in Eq. (56), we get

$$
\begin{aligned}
\delta u_{j}^{\text {eff }}= & \sigma^{2}(2 \pi)^{d / 2} \cdot \prod_{i=1}^{d} l_{i} \int_{0}^{\infty} d t^{\prime} \int_{\mathbf{q}} \mathrm{i} q_{j} \mathbf{q}^{2} \exp \left[-\left(q_{i} l_{i}\right)^{2}\right] \\
& \underbrace{\exp \left[-\left(D \mathbf{q}^{2}-\mathbf{i} \overline{\mathbf{u}}\right) t^{\prime}\right]}_{f} .
\end{aligned}
$$

We perform the integration over the Fourier variables followed by the time integration. However, the time integration can not be carried out explicitly. Therefore, we choose a twofold approach. We first evaluate the integral numerically and secondly we expand the integrals in orders of small inverse Peclet numbers. The latter assumption is limited to advectively dominated transport regimes.

In the sequel, we will use the following notations:

$$
\bar{\sigma}^{2}=\frac{\sigma^{2}}{\bar{u}_{1}^{2} l_{1}^{2}}, \quad \beta=l_{2} / l_{1}, \quad \alpha=\bar{u}_{2} / \bar{u}_{1},
$$

where $l_{i}$ and $\sigma^{2}$ are the correlation lengths and the variance of the function (32), respectively, and $u_{i}$ are the mean drift defined in Eq. (45). In the discussion which follows, we will keep the variables $l_{1}=\bar{l}$ and $\bar{u}_{1}=\bar{u}$ fixed. In order to be consistent with our previous expansion in small inverse Peclet numbers, we assume $l_{2} \geqslant l_{1}$ and $\bar{u}_{2} \geqslant \bar{u}_{1}$ so that $D /\left(\bar{u}_{2} l_{2}\right)$ $\leqslant D /\left(\bar{u}_{1} l_{1}\right)$.

\section{Numerical evaluation}

The results of the numerical evaluation of Eq. (57) are plotted for $\delta u_{1}^{\text {eff }}$ and $\delta u_{2}^{\text {eff }}$ with $\alpha=1$ and different parameters $\beta$ in Fig. 1.

The smaller $\beta$, the larger is $\delta u_{2}^{\text {eff }} / u_{2}$. On the other hand, the smaller $\beta$, the smaller is $\delta u_{1}^{\text {eff }} / u_{1}$. In particular, for $\beta$ smaller than 1 , the $\delta u_{1}^{\text {eff }} / u_{1}$ show a local maximum and the same $\delta u_{1}^{\text {eff }} / u_{1}$ might be caused by particles with different diffusion coefficients. This is a situation one might avoid for particle separation. Therefore, we restrict the further analysis to values of $\beta$ which are equal to or larger than one. For small inverse Peclet numbers the curve for $\delta u_{1}^{\text {eff }} / u_{1}$ shows an almost linear dependence on $\varepsilon$ which is demonstrated in more detail in Fig. 2.

Making use of the results of the numerical evaluation we now choose a transport velocity large enough for the transport to be in the regime of large Peclet numbers. The assumption of large Peclet numbers allows us to expand $\delta u_{j}^{\text {eff }}$ in orders of small inverse Peclet numbers, $\varepsilon \equiv D /\left(\bar{u}_{1} l_{1}\right)$,
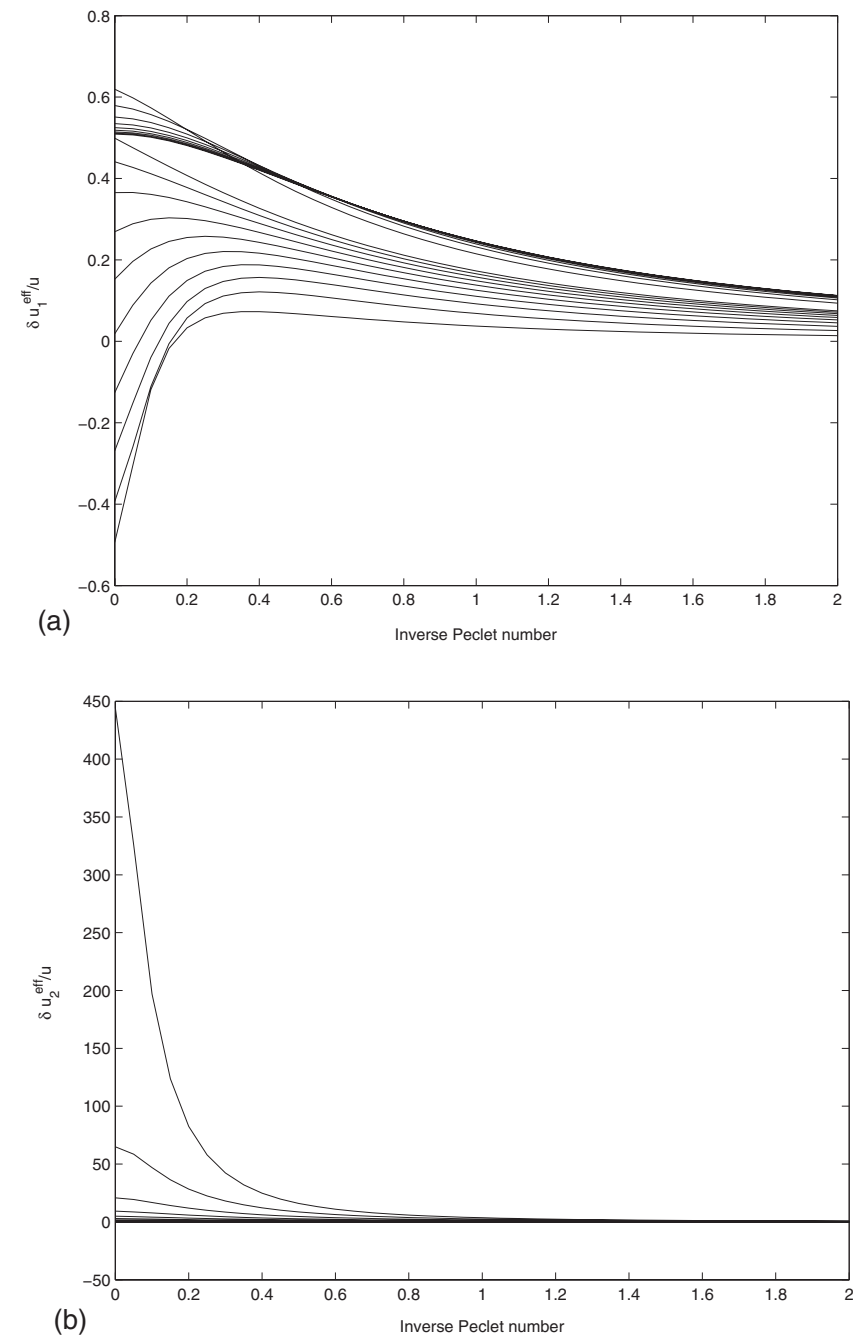

FIG. 1. $\delta u_{1}^{\text {eff }} / u_{1}$ (a) and $\delta u_{2}^{\text {eff }} / u_{2}$ (b) vs inverse Peclet number for different $\beta$ and $\alpha=1$.

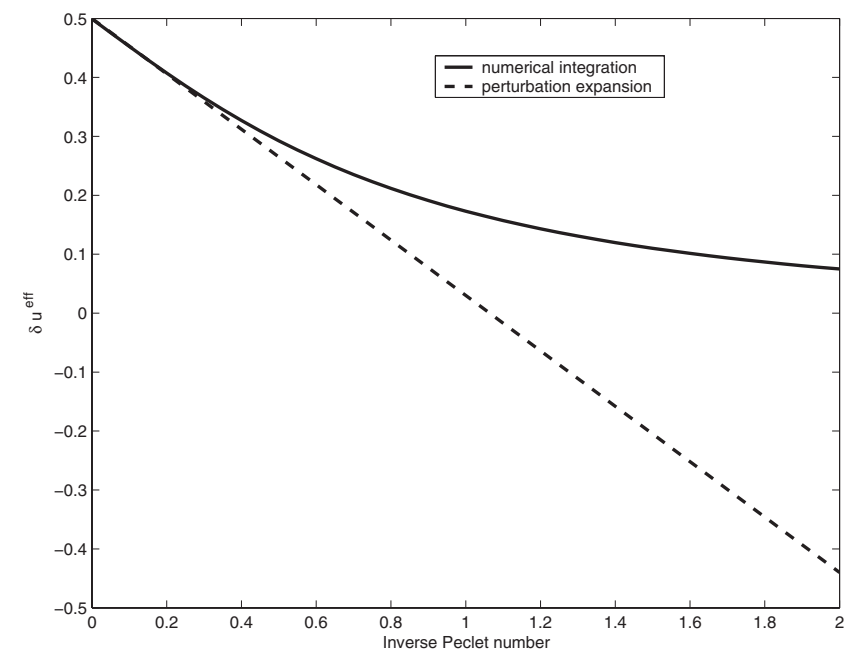

FIG. 2. Comparison of numerical integration of $\delta u_{1}^{\text {eff }} / u_{1}$ and the analytical result for small inverse Peclet numbers vs inverse Peclet number for $\beta=1$. 


$$
\delta u_{j}^{\mathrm{eff}}=\delta u_{j}^{\mathrm{eff}, 0}+\varepsilon \delta u_{j}^{\mathrm{eff}, 1}+O\left(\varepsilon^{2}\right) .
$$

We therefore first expand the integrand $f$ with respect to small inverse Peclet number $f(\varepsilon)=f(0)+\varepsilon f^{\prime}(0)+\mathcal{O}\left(\varepsilon^{2}\right)$, where $\varepsilon=D /(u \bar{l})$ and $\bar{u}=|\overline{\mathbf{u}}|$ denotes the norm of $\overline{\mathbf{u}}$. We define $\bar{u}:=\bar{u}_{1}$ and $\bar{l}:=\bar{l}_{1}$. This series reads

$$
f(\varepsilon)=e^{\left(\varepsilon \mathbf{q}^{2}-i \overline{\mathbf{u}} \mathbf{q} \bar{u} \bar{l}\right) u \bar{l} t^{\prime}}=e^{(i \overline{\mathbf{u}}) t^{\prime}}+\varepsilon\left(\mathbf{q}^{2} \bar{u} \bar{l} t^{\prime}\right) e^{(i \overline{\mathbf{u}} \mathbf{q}) t^{\prime}}+\mathcal{O}\left(\varepsilon^{2}\right) .
$$

Inserting this expression in the equation for $\delta u_{j}^{\text {eff }}(\infty)$ reads

$$
\delta u_{j}^{\text {eff }}=\delta u_{j}^{\text {eff, } 0}(\infty)+\varepsilon \delta u_{j}^{\text {eff, } 1}(\infty)+\mathcal{O}\left(\varepsilon^{2}\right) .
$$

This latter series is then truncated after the two first terms and integrated explicitly. The explicit results are compared with results obtained from numerical evaluation of the time integration.

\section{Infinite Peclet numbers, $\varepsilon=0$}

In case of infinite Peclet numbers, we obtain the zero order term of the expansion (60) and we evaluate for $d=2$, $j=1,2$ the integral

$$
\begin{aligned}
\delta u_{j}^{\mathrm{eff}, 0}= & \sigma^{2}(2 \pi)^{d / 2} \prod_{i=1}^{d} l_{i} \int_{0}^{\infty} d t^{\prime} \int_{\mathbf{q}} \mathrm{i} q_{j} \mathbf{q}^{2} \\
& \times \exp \left(-\left(q_{i} l_{i}\right)^{2} / 2\right) \exp \left\{(\mathrm{i} \overline{\mathbf{u}} \mathbf{q}) t^{\prime}\right\} .
\end{aligned}
$$

We obtain

$$
\begin{aligned}
& \delta u_{1}^{\mathrm{eff}, 0}=-\bar{\sigma}^{2} \bar{u}_{1} \frac{\beta^{4}-\alpha^{2}+3 \beta^{2} \alpha^{2}+\beta^{2}}{2\left(\alpha^{2}+\beta^{2}\right)^{2}}, \\
& \delta u_{2}^{\mathrm{eff}, 0}=-\bar{\sigma}^{2} \bar{u}_{2} \frac{\alpha^{2}-\beta^{4}+3 \beta^{2}+\beta^{2} \alpha^{2}}{2\left(\alpha^{2}+\beta^{2}\right)^{2} \beta^{2}} .
\end{aligned}
$$

We next discuss the influence of the parameters (correlation lengths and mean velocities) defining the "zeros" order effective velocity corrections, on the effective drifts. Choosing the parameter $\beta$ equal to one, the medium becomes isotropic and the velocity components reduce to

$$
\delta u_{1}^{\text {eff, } 0}=-\bar{\sigma}^{2} \bar{u}_{1} \frac{1}{\left(1+\alpha^{2}\right)}, \quad \delta u_{2}^{\text {eff, } 0}=-\bar{\sigma}^{2} \bar{u}_{2} \frac{1}{\left(1+\alpha^{2}\right)} .
$$

Adding $\overline{\mathbf{u}}$ and the zero order correction term $\delta \mathbf{u}^{\text {eff, } 0}$ together gives an effective drift which is parallel to the mean drift. No deviation due to the heterogeneity are induced. We will find an analogous result adding the higher order term $\delta \mathbf{u}^{\text {eff, } 1}$. It demonstrates that in an isotropic medium, an irrotational flow does not induce a modification of the direction of the effective velocity field.

On the other hand, for largely anisotropic media, $\beta$ $\rightarrow \infty$ we find

$$
\delta u_{1}^{\mathrm{eff}, 0}=-\sigma^{2} \frac{\bar{u}_{1}}{2}, \quad \delta u_{2}^{\mathrm{eff}, 0}=0 .
$$

In this situation we have a perfectly layered medium in which the particles move only parallel to the layers. For $\alpha$ $\rightarrow \infty$ the velocity coefficients $\delta u_{i}^{\text {eff, }, 0}$ tend to zero and we have again a layered medium. We will exclude these limit case and study $1 \leqslant \alpha, \beta \leqslant M$. To study more deeply the influences of the heterogeneities (the correlations length of the medium) on the effective velocity, we fix the mean drift $\bar{u}_{1}=\bar{u}_{2}$, i.e., we set $\alpha=1$ and further set $M=2$,

$$
\begin{aligned}
& \delta u_{1}^{\mathrm{eff}, 0}=-\bar{\sigma}^{2} \bar{u}_{1} \frac{\beta^{4}-1+4 \beta^{2}}{2\left(1+\beta^{2}\right)^{2}}, \\
& \delta u_{2}^{\mathrm{eff}, 0}=-\bar{\sigma}^{2} \bar{u}_{2} \frac{1-\beta^{4}+4 \beta^{2}}{2\left(1+\beta^{2}\right)^{2} \beta^{2}} .
\end{aligned}
$$

In Fig. 4.2.2, the velocity components $\delta u_{i}^{\text {eff, } 0}$ are plotted for different $\beta$ ranging from 1 to 2 with a variance $\bar{\sigma}^{2}=0.5$. We recall that the variation of $\beta$ means a variation of $l_{2}$, since $l_{1}$ is kept fixed. We see that due to the zero order terms $\delta u_{i}^{\text {eff, } 0}$, the effective drift $u_{i}^{\text {eff }}$ will be decreased against the local mean drift $\bar{u}_{i}$.

\section{First order correction, $\mathcal{O}(\varepsilon)$}

We integrate $\delta u_{j}^{\text {eff, } 1}$ given in Eq. (61) for $d=2, j=1,2$,

$$
\begin{aligned}
\delta u_{j}^{\mathrm{eff}, 1}= & \sigma^{2}(4 \pi)^{d / 2} \prod_{i=1}^{d} l_{i} \int_{0}^{\infty} d t^{\prime} \int_{\mathbf{q}} \mathrm{i} q_{j} \mathbf{q}^{2} \\
& \times \exp \left(-\left(q_{i} l_{i}\right)^{2}\right) \exp \left\{+\mathrm{i} \overline{\mathbf{u}} \mathbf{q} t^{\prime}\right\} \mathbf{q}^{2} \bar{u} \bar{l} t^{\prime},
\end{aligned}
$$

and we get for the velocity components

$$
\begin{aligned}
& \delta u_{1}^{\mathrm{eff}, 1}=\frac{3 \bar{u}_{1}}{4} \sqrt{\pi} \bar{\sigma}^{2} \beta \frac{6 \alpha^{2} \beta^{2}-4 \alpha^{4}+5 \alpha^{4} \beta^{2}+\beta^{2}-4 \alpha^{2}}{\left(\beta^{2}+\alpha^{2}\right)^{7 / 2}}, \\
& \delta u_{2}^{\mathrm{eff}, 1}(\infty)=-\frac{3 \bar{u}_{2}}{4} \sqrt{\pi} \bar{\sigma}^{2} \beta \frac{4 \beta^{2}-6 \alpha^{2}-5+4 \alpha^{2} \beta^{2}-\alpha^{4}}{\left(\beta^{2}+\alpha^{2}\right)^{7 / 2}} .
\end{aligned}
$$

Again, we discuss the influence of the parameters (correlation lengths and mean velocities) defining the first order effective velocity corrections, on the effective drifts. In extremely anisotropic media, $\beta \rightarrow \infty$, both first order velocity components approach zero. In isotropic media, $\beta=1$, the results of the first order velocity components reduce to

$$
\begin{aligned}
& \delta u_{1}^{\mathrm{eff}, 1}=\frac{3 \bar{u}_{1}}{4} \sqrt{\pi} \bar{\sigma}^{2}\left(1+\alpha^{2}\right)^{-3 / 2}, \\
& \delta u_{2}^{\mathrm{eff}, 1}=\frac{3 \bar{u}_{2}}{4} \sqrt{\pi} \bar{\sigma}^{2}\left(1+\alpha^{2}\right)^{-3 / 2},
\end{aligned}
$$

indicating again that no deviation in transverse direction occurs since all particles stay on the same flow line. However, the particles move with different velocities depending on their molecular weights or diffusion coefficients. To this end, isotropic medium may separate particles in time. In such medium, larger particles will be less retarded than smaller ones.

Choosing the parameter $\alpha$ equal to one, the expressions for the first order drift components simplify to 


$$
\begin{aligned}
& \delta u_{1}^{\mathrm{eff}, 1}=\frac{3 \bar{u}_{1}}{4} \sqrt{\pi} \bar{\sigma}^{2} \beta \frac{12 \beta^{2}-8}{\left(\beta^{2}+1\right)^{7 / 2}}, \\
& \delta u_{2}^{\mathrm{eff}, 1}=-\frac{3 \bar{u}_{2}}{4} \sqrt{\pi} \bar{\sigma}^{2} \beta \frac{8 \beta^{2}-12}{\left(\beta^{2}+1\right)^{7 / 2}},
\end{aligned}
$$

which are plotted in Fig. 2, for different $\beta$ ranging from 1 to 2 , while holding $\bar{u}_{1}$ and $l_{1}$ fixed. The variance $\bar{\sigma}^{2}$ is again set to 0.5 . We see that in this situation, the effective drifts $u_{1}^{\text {eff,i }}$ can induce a deviation in transverse direction. Owing to Eq. (61) it is therefore possible to obtain effective velocities whose directions depend on the diffusion constants (or molecular weights).

\section{NUMERICAL STUDY}

In Sec. IV, we have seen that the effective drifts depend on the small scale diffusion coefficient in compressible flows [see Eq. (61)]. In this section we perform numerical experiments to illustrate the aformentioned effects. We consider a transport problem (advection-diffusion) with heterogeneous compressible velocities and study numerically the large scale effective drift. Taking an initial point-like solute, we compare the trajectories when varying the diffusion constants. We emphasize that in compressible flows the center of mass of the solute does not depend on the diffusion coefficient (see Ref. 13 for similar numerical computation in this latter case).

We consider the transport equation in a reference domain $\Omega=(0,1)^{2}$,

$$
\begin{aligned}
& \frac{\partial c}{\partial t}+\nabla[\mathbf{u}(x) c]=D \nabla^{2} c(x, t) \in \Omega \times[0, T], \\
& c(x, 0)=c_{0}(x), \quad c(x, t)=0 \text { for } x \in \partial \Omega,
\end{aligned}
$$

where the velocity field is split in $\mathbf{u}=\overline{\mathbf{u}}+\widetilde{\mathbf{u}}$, where $\overline{\mathbf{u}}$ is a constant mean and where we suppose that the random compressible velocity field $\widetilde{\mathbf{u}}$ is defined by a zero mean random Gaussian correlation function according to Eq. (32),

$$
\begin{aligned}
\overline{\widetilde{\mathbf{u}}_{i}\left(\mathbf{x}_{1}\right) \widetilde{\mathbf{u}}_{j}\left(\mathbf{x}_{2}\right)} & =\partial_{i} \partial_{j} \sigma^{2}(2 \pi)^{1 / 2} \exp \left[-\sum_{k=1}^{2} \frac{\left(x_{1 k}-x_{2 k}\right)^{2}}{2 l_{k}^{2}}\right] \\
& =C_{i j}\left(\mathbf{x}_{1}, \mathbf{x}_{2}\right),
\end{aligned}
$$

where $l_{k}, k=1,2$ are the correlation lengths in the $\mathbf{x}_{1}, \mathbf{x}_{2}$ $\in \mathbb{R}^{2}$ directions, respectively, and $\sigma^{2}$ is the variance.

Such a potential can be generated by a superposition of randomly chosen cosine modes ${ }^{25}$ as follows. Define

$$
\mathbf{u}_{i}^{N}=\sigma \sqrt{\frac{2}{N}} \sum_{j=1}^{N} k_{i}^{j} \cos \left(\mathbf{k}^{j} \mathbf{x}+\omega_{j}\right),
$$

where each component of $\mathbf{k}^{j}=\left(k_{1}^{j}, k_{2}^{j}\right)$ obeys a Gaussian distribution with zero mean and variance given by $1 / l_{i}^{2}$. Due to the central limit theorem, $\mathbf{u}_{\mathbf{i}}=\lim _{N \rightarrow \infty} \mathbf{u}_{i}^{N}$ is Gaussian and it can be shown (see, for example, Ref. 21, and the references therein) that the limit correlation function is given by Eq. (75), or by Eq. (32) in Fourier space.

For the simulation, we transform the transport equation (73) by the method of lines in a system of ordinary differen-
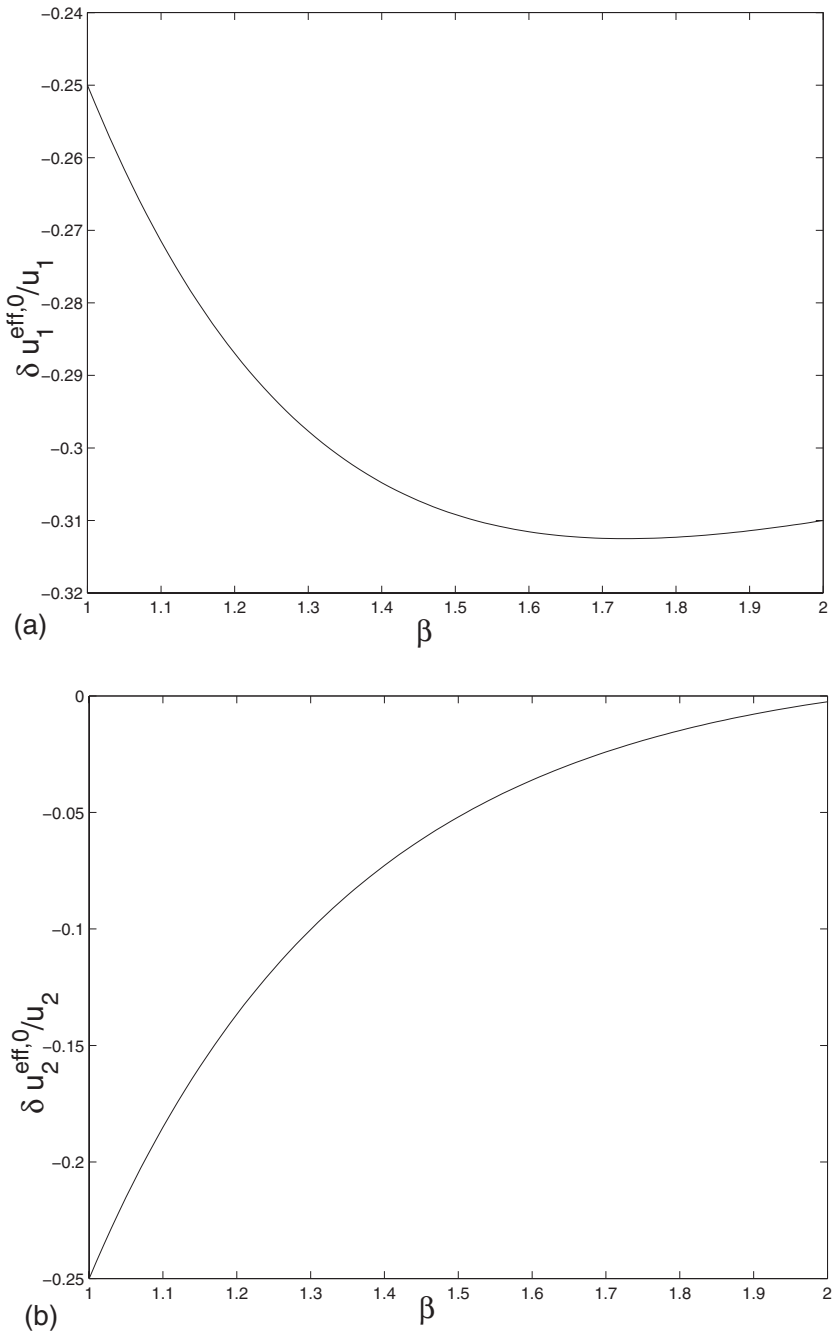

FIG. 3. $\delta u_{1}^{\mathrm{eff}, 0} / u_{1}$ (a) and $\delta u_{2}^{\mathrm{eff}, 0} / u_{2}$ (b) vs $\beta$.

tial equations which are solved using the orthogonal RungeKutta Chebyshev method ROCK4 ${ }^{26,27}$ We discretize $\Omega$ with mesh-size $1 / 400$ in each spatial direction $\mathbf{x}_{1}, \mathbf{x}_{2}$. To minimize the influence of artificial boundaries conditions (which we take as zero Dirichlet), we perform the transport simulation on a subdomain $\Omega_{s}=[0.0650,0.9375] \times[0.0650,0.9375]$. For all the simulations, the initial concentration and the final time are the same. The initial concentration at time $t=0$ is given by $c_{0}(x)=\chi_{D}(x)$, where $\chi_{D}(x)=1$ for $x \in D$ and zero for $x \in \Omega \backslash D$ where $D=[0.0650,0.090] \times[0.0650,0.090]$. The transport simulations are stopped at time $t=0.9$.

In the following we study three situations: We fix a reference correlation length $l_{1}=\bar{l}=1 / 10$, set $\alpha=1$ and consider $\beta=1, \beta \simeq 1.43$ and $\beta=2$. In all the simulations we set $\bar{u}_{1}$ $=\bar{u}_{2}=0.2$ and $\bar{\sigma}=0.5$. Thus, the variance of the correlation function (75) is $\sigma^{2}=0.01$. We simulate the transport equation (73) with two different diffusion constants $D=0.001$ and $D$ $=0.005$. The Peclet numbers satisfy $4 \leqslant \mathrm{Pe} \leqslant 20$ and we are therefore in the advection dominated regime.

Our theoretical results predict a ratio $a \equiv u_{2}^{\text {eff }} / u_{1}^{\text {eff }}$ of

$$
\begin{aligned}
& a=1.18, \quad \beta=1.43, \quad \mathrm{Pe}=4 \text {, } \\
& a=1.31, \quad \beta=1.43, \quad \mathrm{Pe}=20,
\end{aligned}
$$



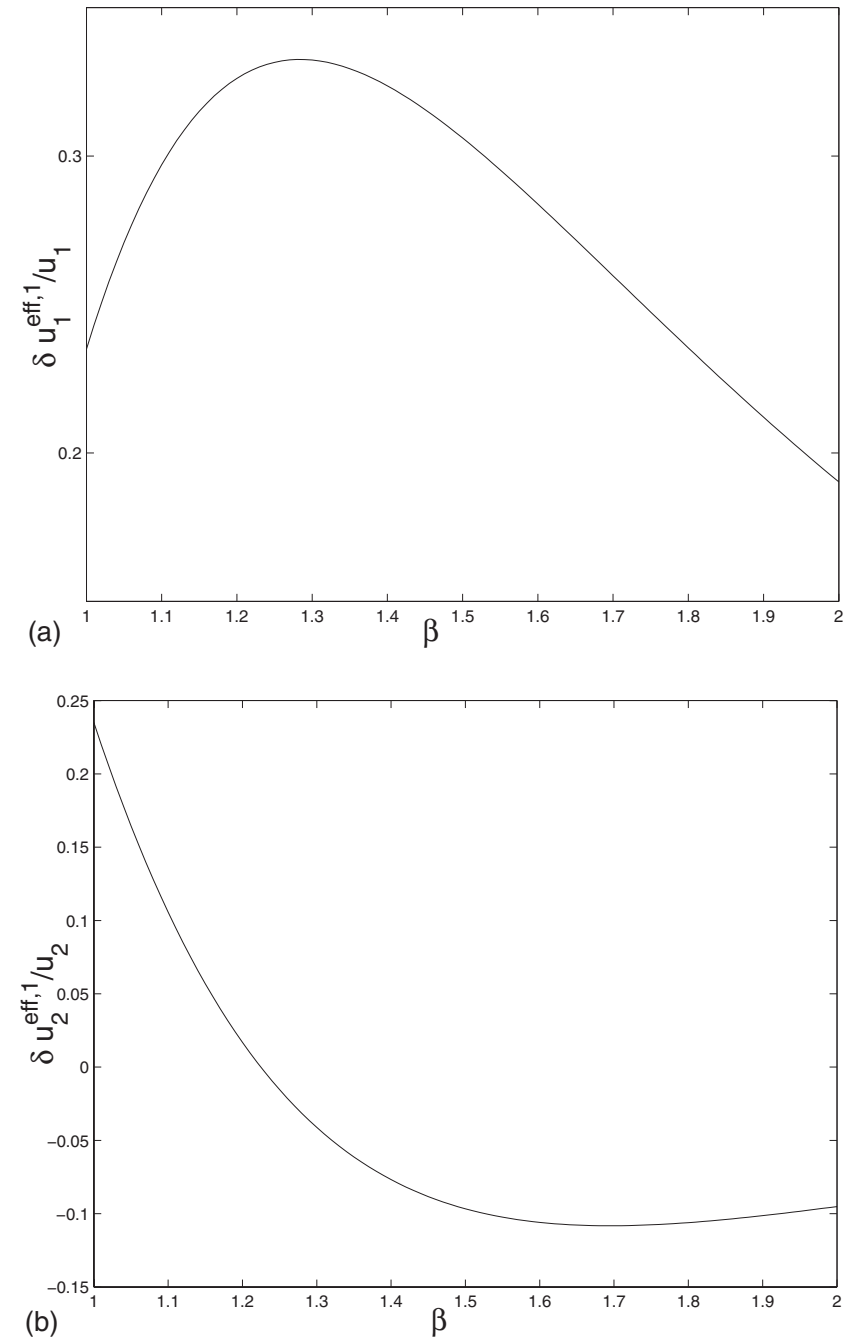

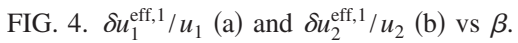

$$
\begin{aligned}
& a=1.32, \quad \beta=2.00, \quad \mathrm{Pe}=4, \\
& a=1.39, \quad \beta=2.00, \quad \mathrm{Pe}=20 .
\end{aligned}
$$

For two different Peclet numbers the ratio $a$ differs mostly for $\beta=1.43$. In other words, separation is highest for $\beta$ $=1.43$ compared with $\beta=1$ and $\beta=2$. This is confirmed by numerical simulations.

In the first simulation, we choose $l_{2}=l_{1}$ and we see in Fig. 3 that the effective velocity is the same for the two different diffusion constants. This agrees with the results obtained in Sec. IV B 2.

In the second simulation, we choose $l_{2}=l_{1} / 0.7$, i.e., $\beta$ $\simeq 1.43$. According to the results of Sec. IV B 3 we should observe an effective drift depending on the diffusion constant. Indeed, we observe in Fig. 4 that molecules with different diffusion constants migrate to different locations of the medium and thus are separated.

Finally, we perform a simulation with $l_{2}=2 \cdot l_{1}$, i.e., $\beta$ $=2$. According to the results of Sec. IV B 3 this situation is less favorable for a weight-based separation of particles since for large $\beta$ both, the first order effective velocities $\delta u_{1}^{\text {eff,i }}(\infty) i=1,2$ tend to zeros. We see in Fig. 5 that no deflection of the center of mass of the particles can be ob-
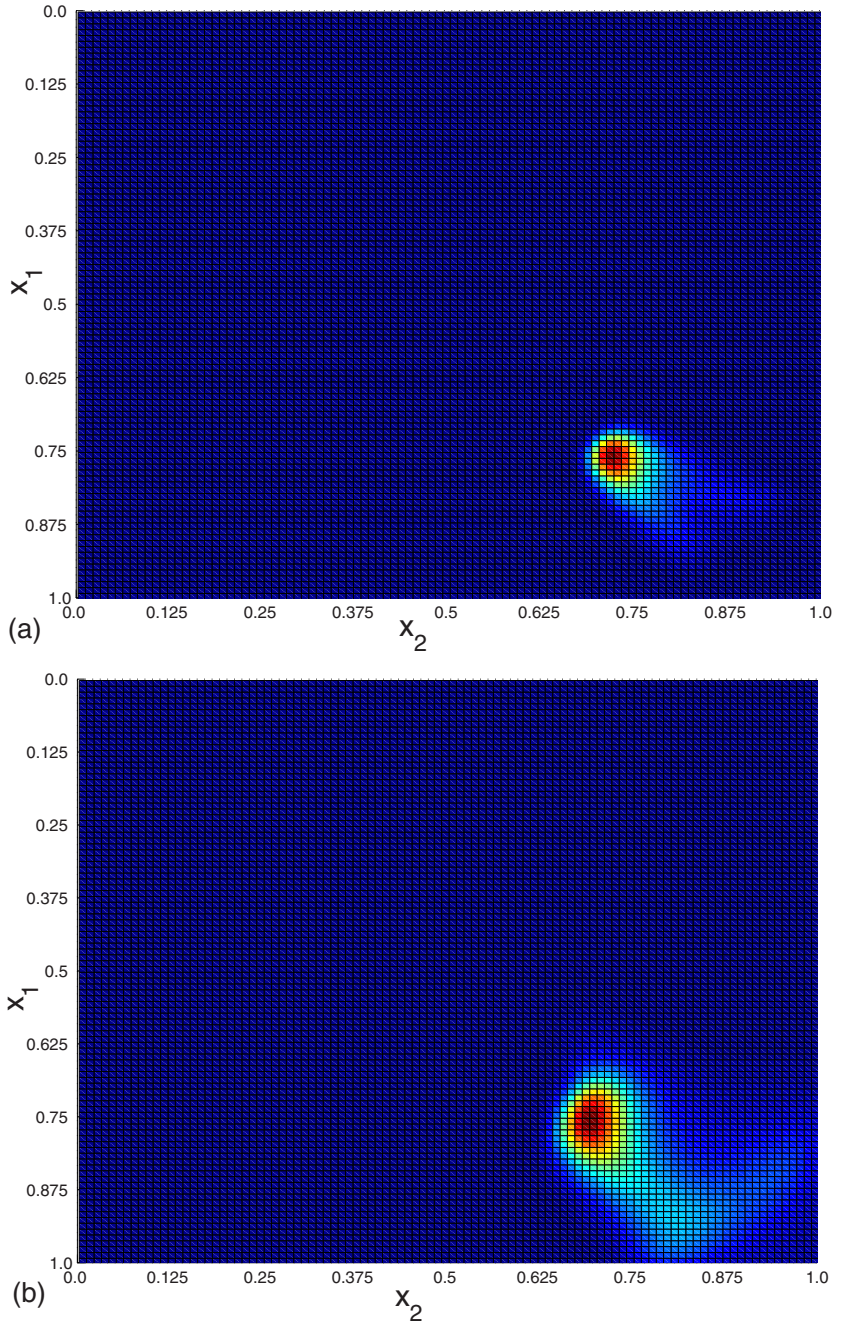

FIG. 5. (Color online) Transport simulation in isotropic medium $(\beta=1)$ with $D=0.001$ (a) and $D=0.005$ (b).

served. But compared to the isotropic situation (Fig. 3), we see that the strong heterogeneities chosen in this case increase the mixing of the particles (see Figs. 6 and 7).

\section{CONCLUSIONS}

We have studied the large scale or effective transport velocity for particles in heterogeneous compressible flows with mean drift. We have shown that such flows can substantially modify the transport behavior generating a large scale drift which differs from the local mean drift. This result implies a major difference between transport in compressible and incompressible flows with mean drift. For transport in incompressible flows the large scale drift of the dissolved particles equals the local mean drift. Heterogeneous fluid velocities have an impact on large scale mixing only. For transport in compressible flows, however, large scale drift as well as large scale dispersion coefficients are modified against their local values. Moreover, we completed the work of Vergassola and Avellaneda. ${ }^{14}$ There, Vergassola and Avellaneda proved that heterogeneously distributed compressible flows with vanishing mean drift do not generate any heterogeneity induced large scale drift (or ballistic transport). Our paper 

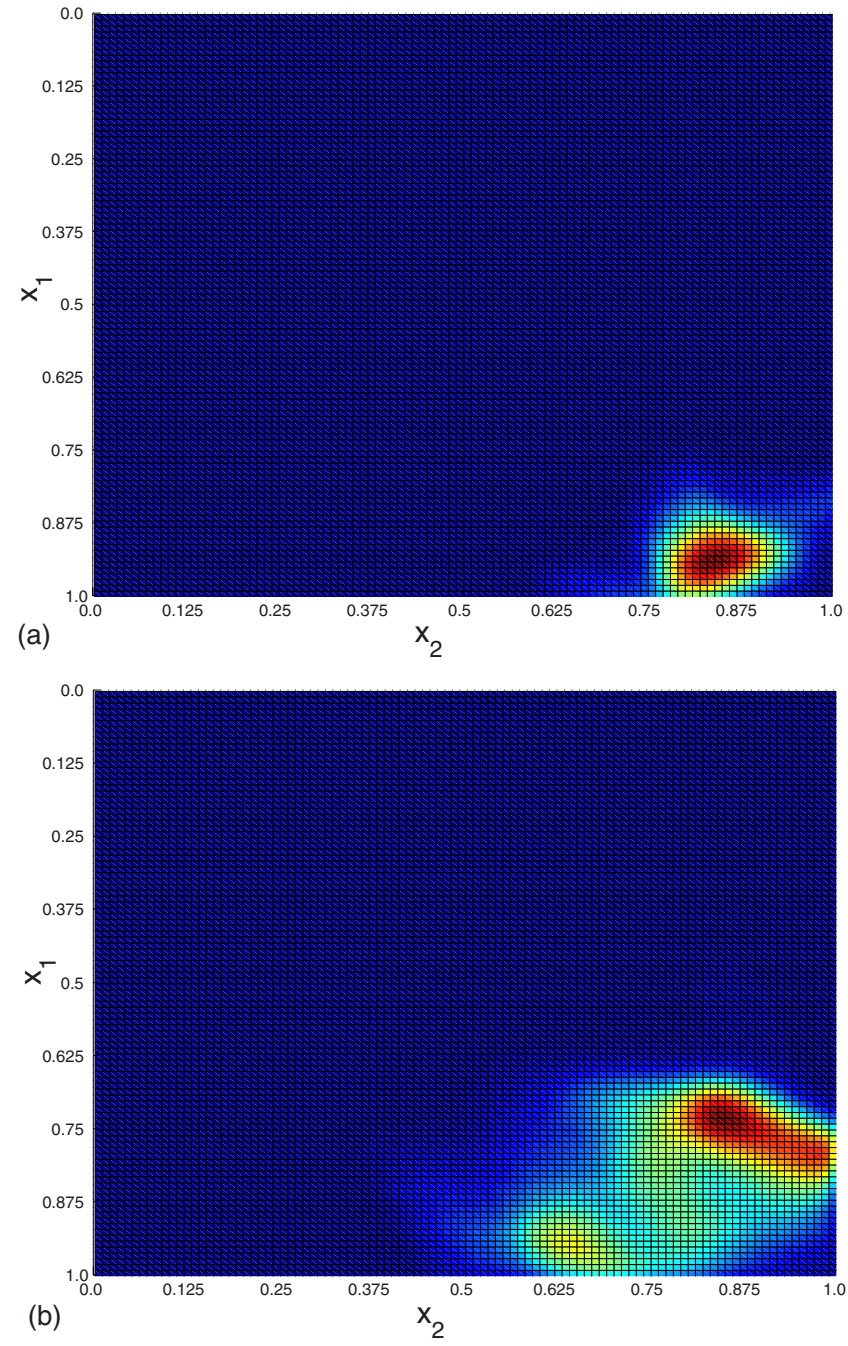

FIG. 6. (Color online) Transport simulation in anisotropic medium $(\beta$ $\simeq 1.43$ ) with $D=0.001$ (a) and $D=0.005$ (b).

demonstrates that ballistic transport might be generated but only in the case of particle transport in compressible flows with nonvanishing mean drift.

For a class of flow fields given by Gaussian stationary random processes, we derived explicit formulas for the large scale drift making use of perturbation theory. These explicit results allow us to quantify the influence of the heterogeneities upon the effective transport velocity. In the case of advection dominated transport, the effective drift is decreased against the local mean drift. Moreover, its value depends on the local diffusion coefficients and thus on the molecular weights of the transported particles. This result might be of interest for the realization of efficient weight-based separation devices. ${ }^{17-19}$ To quantify the efficiency of such a device the large scale dispersive movement in particular the increased transverse mixing of particles might impact and eventually hinder the separation of particles. The quality of particle separation is determined by the distance between two neighboring particles clouds with different diffusion coefficient or molecular weights, $\overline{\Delta x}=\Delta u t \approx u^{\text {eff, } 1} \varepsilon t$, and the spreading width of the particle cloud $\sqrt{2 D^{\text {eff }}} t$. For long times or times much larger than the dispersive time scale $l^{2} / D, \overline{\Delta x}$
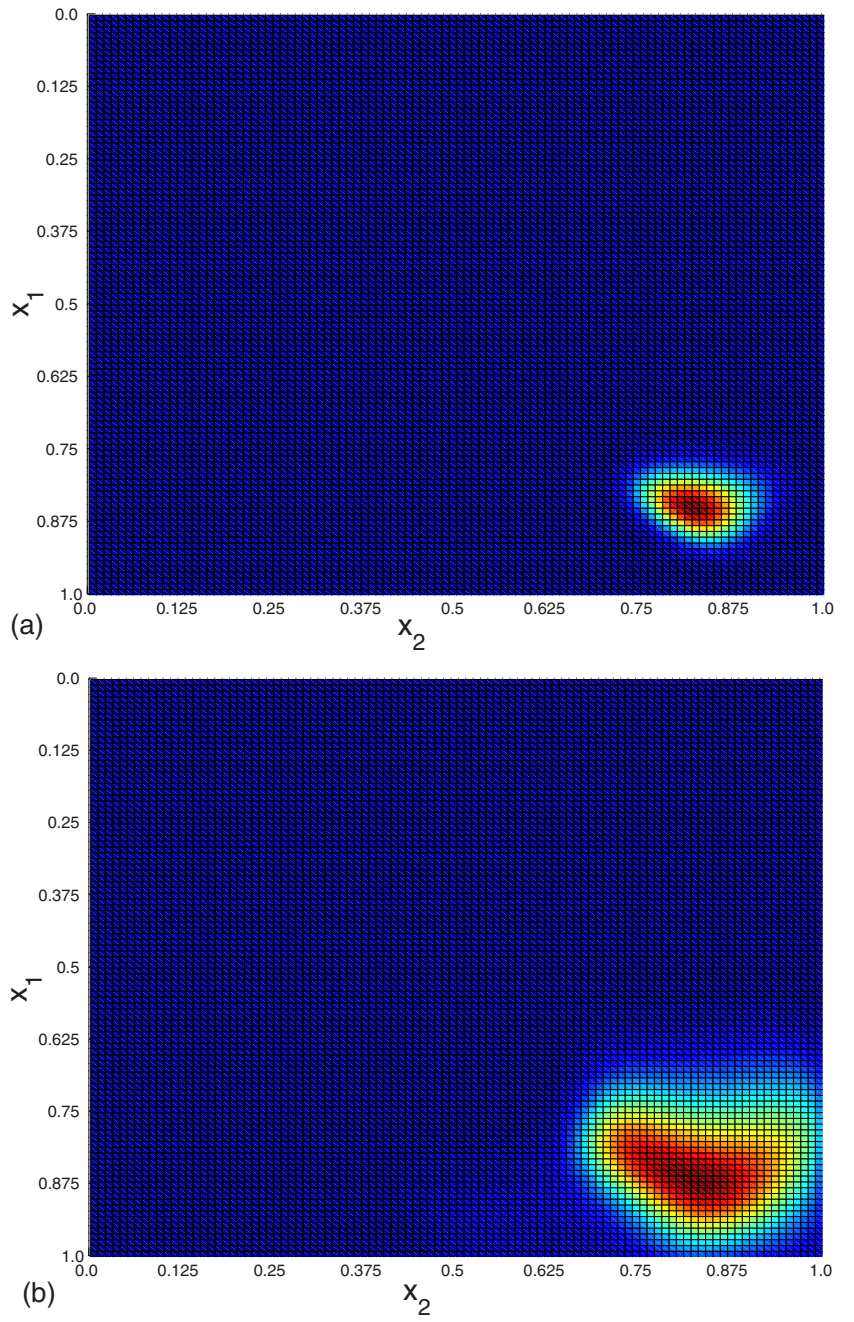

FIG. 7. (Color online) Transport simulation in anisotropic medium $(\beta=2)$ with $D=0.001$ (a) and $D=0.005$ (b).

dominates the dispersive spreading $\sqrt{2 D^{\text {eff }} t}$ since the first scales proportional to time, whereas the second grows only proportional to the square root of time. To this end, particle separation will always take place in the long time limit or $t$ $\gg l^{2} / D$. Designing a sorting device, however, requires devices of finite size and thus the consideration of preasymptotic effects as well. For times smaller than $l^{2} / D$, the effective dispersion coefficient can be approximated by its local value as known from Dentz and co-workers. ${ }^{21}$ In this case, separation requires $\overline{\Delta x}=\Delta u t \approx u^{\mathrm{eff}, 1} \varepsilon t>\sqrt{2 D t}$ or $t$ $>u / u^{\text {eff, } 1} l^{2} / D$. To this end, $u^{\text {eff, } 1}$ has to be chosen to be much larger than $u$ to provide good sorting conditions. The design and optimization of a sorting device and testing its performance under realistic conditions will be studied in the future. In this paper, we give the general proof of our concept.

\section{ACKNOWLEDGMENTS}

The work of Assyr Abdulle is supported by an EPSRC Advanced Fellowship, Grant No. EP/E05207X/1.

${ }^{1}$ S. Whitaker, The Method of Volume Averaging (Kluwer, Dordrecht, 1999). ${ }^{2}$ J. W. Haus and K. W. Kehr, "Diffusion in regular and disordered lattices," Phys. Rep. 150, 263 (1987). 
${ }^{3}$ J. P. Bouchaud and A. Georges, "Anomalous diffusion in disordered media: Statistical mechanisms, models and physical applications," Phys. Rep. 195, 127 (1990).

${ }^{4}$ G. Dagan, Flow and Transport in Porous Formations (Springer-Verlag, New York, 1989).

${ }^{5}$ L. W. Gelhar, Stochastic Subsurface Hydrology (Prentice-Hall, Englewood Cliffs, NJ, 1993).

${ }^{6}$ D. W. McLaughlin, G. C. Papanicolaou, and O. R. Pironneau, "Convection of microstructure and related problems," SIAM J. Appl. Math. 45, 780 (1985).

${ }^{7}$ M. Avellaneda and A. J. Majda, "An integral representation and bounds on the effective diffusivity in passive advection by laminar and turbulent flows," Commun. Math. Phys. 138, 339 (1991).

${ }^{8}$ A. J. Majda and P. R. Kramer, "Simplified models for turbulent diffusion: Theory, numerical modelling, and physical phenomena," Phys. Rep. 314, 237 (1999).

${ }^{9}$ W. E., "Homogenization of linear and nonlinear transport equations," Commun. Pure Appl. Math. 45, 3 (1992).

${ }^{10}$ A. J. Majda and R. M. MacLaughlin, "The effect of mean flows on enhanced diffusivity in transport by incompressible periodic velocity fields," Stud. Appl. Math. 89, 245 (1993).

${ }^{11}$ G. A. Pavliotis, "Homogenization theory for advection-diffusion equation with mean flow," Ph.D. thesis, Rensselear Polytechnic Institute, Troy, NY (2002).

${ }^{12}$ I. Lunati, S. Attinger, and W. Kinzelbach, "Large scale dispersivities for transport in arbitrary nonuniform flow fields," Water Resour. Res. 38, 10 (2002).

${ }^{13}$ A. Abdulle and A. Attinger, "Homogenization method for transport of DNA particles in heterogeneous arrays," Lect. Notes Comput. Sci. 39, 23 (2004).
${ }^{14}$ M. Vergassola and M. Avellaneda, "Scalar transport in compressible flow," Physica D 106, 1 (1997).

${ }^{15}$ R. M. MacLaughlin and M. G. Forest, "An inelastic, scale-separated model for mixing, with application to atmospheric transport phenomena," Phys. Fluids 11, 4 (1999).

${ }^{16}$ T. Goudon and F. Poupaud, "Homogenization of transport equations: Weak mean field approximation," SIAM J. Math. Anal. 36, 3 (2005).

${ }^{17}$ T. A. Duke and R. H. Austin, "Microfabricated sieve for the continuous sorting of macromolecules," Phys. Rev. Lett. 80, 1552 (1998).

${ }^{18}$ D. Ertas, "Lateral separation of macromolecules and polyelectrolytes in microlithographic arrays," Phys. Rev. Lett. 80, 1548 (1998).

${ }^{19}$ L. R. Huang, P. Silberzan, J. O. Tegenfeldt, E. C. Cox, J. C. Sturm, R. H. Austin, and H. Craighead, "Role of molecular size in ratchet fractionation," Phys. Rev. Lett. 89, 178301 (2002).

${ }^{20} \mathrm{P}$. M. Morse and H. Feshbach, Methods of Theoretical Physics (McGrawHill, New York, 1953).

${ }^{21}$ M. Dentz, H. Kinzelbach, S. Attinger, and W. Kinzelbach, "Numerical studies of the transport behavior of a passive solute in a two-dimensional incompressible random flow field," Phys. Rev. E 67, 046306 (2003).

${ }^{22} \mathrm{M}$. Dentz, "Temporal behavior of transport parameters in heterogeneous porous media," Ph.D. thesis, University of Heidelberg (2000).

${ }^{23}$ R. H. Kraichnan, "The structure of isotropic turbulence at very high Reynolds numbers," J. Fluid Mech. 5, 497 (1959).

${ }^{24}$ P. H. Roberts, "Analytical theory of turbulent diffusion," J. Fluid Mech. 11, 257 (1961).

${ }^{25}$ R. H. Kraichnan, "Diffusion by a random velocity field," J. Fluid Mech. 13, 22 (1970).

${ }^{26}$ A. Abdulle, "Fourth order Chebyshev methods with recurrence relation," SIAM SISC 23, 6 (2002).

${ }^{27}$ A. Abdulle, "Multiscale methods for advection-diffusion problems," Discrete Contin. Dyn. Syst., suppl. (2005). 